ESAIM: PROCEEDINGS, November 2002, Vol.12, 67-72

M.Thiriet, Editor

\title{
BIOMECHANICAL SIMULATION FOR SURGERY PLANNING: OSTEOGENIC DISTRACTION OF THE MANDIBLE
}

\author{
Inga HAnsen $^{1,2}$, Robert SchreitTmiller ${ }^{1,3}$ And Horst WAWrzyN $^{4}$
}

\begin{abstract}
In the biomechanical modeling for computer-aided surgery planning in the oral and maxillofacial area the situation at the temporomandibular joint is especially complex and, at the same time, important. We study the effects of a surgical method used for the correction of certain craniofacial deformities, the so-called osteogenic callus distraction. In the case of a median distraction of the mandible, the lower jaw is gradually widened by moving the left and right halves apart from each other (approximately $1 \mathrm{~mm}$ per day). The biomechanical modeling is eventually intended to give insight into the bone loads - stresses, strains and forces - caused by forcing the condylar head of the mandible towards the articular eminence of the temporal bone. Here, we suggest as a first step an approach to represent the distraction process on the mandible and callus regions alone, without consideration of restrictions near the joint.
\end{abstract}

Résumé. Dans la modélisation biomécanique pour la planification chirurgicale assistée par ordinateur il y a des problèmes particulièrement difficiles et, au même temps, importants. Nous étudions les effets d'une intervention chirurgicale permettant d'allonger ou créer de l'os, la distraction osseuse. En cas d'une distraction médiane, la mandibule est fragilisée par une ostéotomie et, après une a période de latence postopératoire qui permet la formation d'un cal précoce, ce cal sera distracté par degrés, a raison de $1 \mathrm{~mm}$ par jour. Le but final de la modélisation biomécanique est d'obtenir une meilleure compréhension des efforts qu' expériencent les os à cause de l' expansion de la mandibule.

Nous proposons comme un premier pas une approche pour représenter le processus de distraction seulement sur les regions de la mandibule et du cal, sans considérer des restrictions près? de la jointure temporo-maxillaire .

\section{INTRODUCTION}

In this paper we present an approach to model the biomechanics of a surgical method applied in cases of congenital deformities or atrophy in bone, when a lengthening of some part of a bone is required. The method is based on a natural mechanism of fracture healing, which occurs when there remains a narrow gap between the two bone fragments. In this situation a cartilaginous substance called fracture callus develops in the gap which is initially relatively compliant and will gradually stiffen and calcify, eventually becoming new bone. It has been found that the callus can be lengthened during this development by applying a mechanical tension continuously, resulting in the formation of more callus and, consequently, a longer section of new bone. We consider here a

\footnotetext{
1 Zentrum Mathematik, Technische Universität München

2 e-mail: hansen@appl-math.tu-muenchen.de

3 e-mail: rschreit@appl-math.tu-muenchen.de

${ }^{4}$ Hightech-Forschungs-Zentrum (Center for Advanced Studies in Maxillofacial Surgery), Technische Universität München; e-mail: HorstWawrzyn@t-online.de
}

(C) EDP Sciences, SMAI 2003 

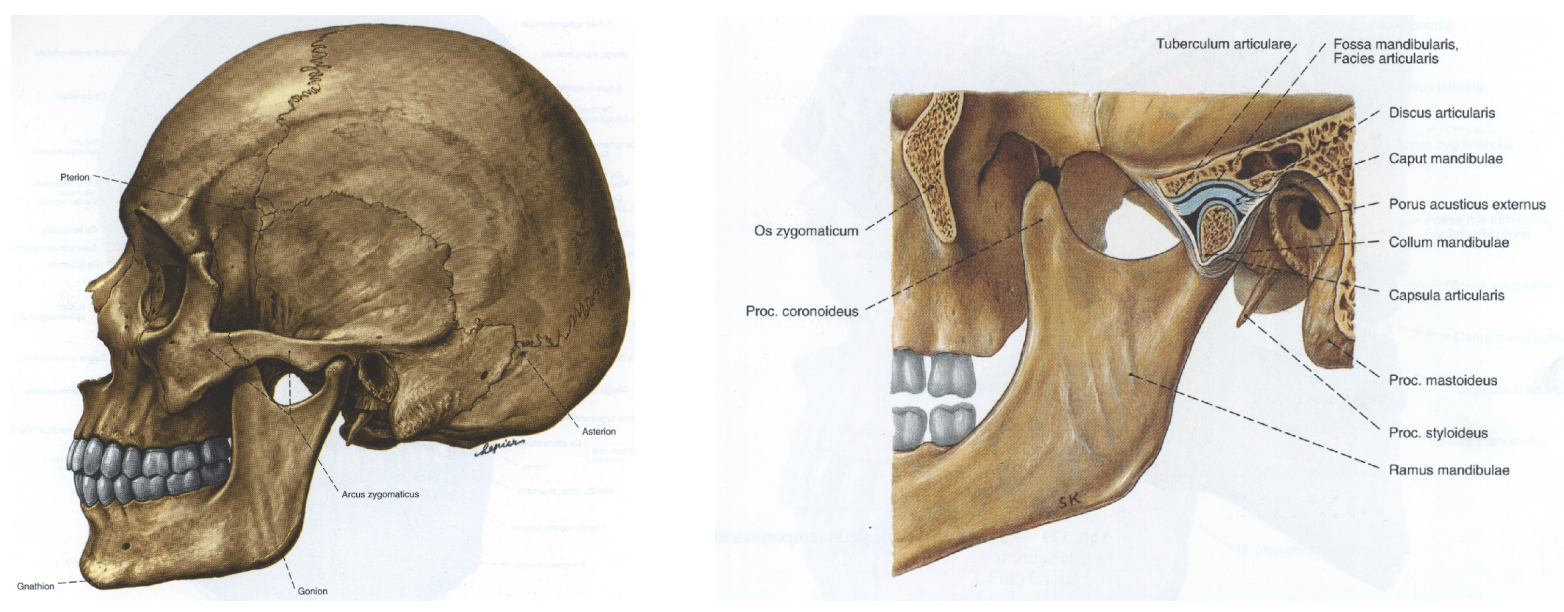

Figure 1. Temporomandibular joint: global view and sagittal cut. The condylar head of the mandible is connected to the temporal bone of the skull by the articular capsule, while the two bony components are separated within the capsule by the articular disc. - From: [6], Figures 63 and 125 .

special surgical procedure from oral and maxillofacial surgery, the median distraction of the mandible, which implies that the lower jaw is widened symmetrically with respect to the median sagittal plane (which separates the left and right halves of the body). An artificial fracture is initially achieved by a surgical operation, and a distractor device, the two ends of which can be moved apart from each other by a screw mechanism connecting them, is attached to or implanted into the two bone ends. After a week, callus material will have formed to bridge the narrow gap left between the bone ends when fixating them with the distractor, and by means of the screw the ends are gradually moved apart from each other in steps of up to $1 \mathrm{~mm}$ per day. (Distraction lengths of more than $2 \mathrm{~cm}$ have been achieved in the mandible.)

The aim of the present study is to model the biomechanics of this procedure - starting on a very simple level. Among the most interesting and important problems in this area is the investigation of the situation at the temporomandibular joint, the joint between the mandible (lower jaw) and the part of the temporal bone surrounding it (see Fig. 1). The condylar head of the mandible can move quite freely in a shallow cavity of the temporal bone, called glenoid fossa. Between these two bony parts of the joint there lies the articular disc, which consists of cartilaginous material. ¿From the point of view of the median distraction, it has to be asked in which way the temporomandibular joints will be affected by the respective displacements of the right and left halves of the mandible.

As a basis for a study of the effects at the condyles, we introduce a finite element model for the distraction without consideration of the temporomandibular joint, i.e. without including the articular disc or the temporal bone and with a free boundary at the condylar surfaces. We thus start after the separation of the two halves of the mandible and formation of a callus bridge between the bone ends and represent the distraction in $n$ time-like steps.

\section{Model}

We assume the median sagittal plane (between left and right, $\{x=0\}$ ) to be a plane of symmetry and consider only one half of the mandible. Further, the distractor device is not represented as implanted into the mandible but thought to be attached to its exterior surface, leading to a straightforward Dirichlet boundary condition for the displacement on the area of its attachment. 


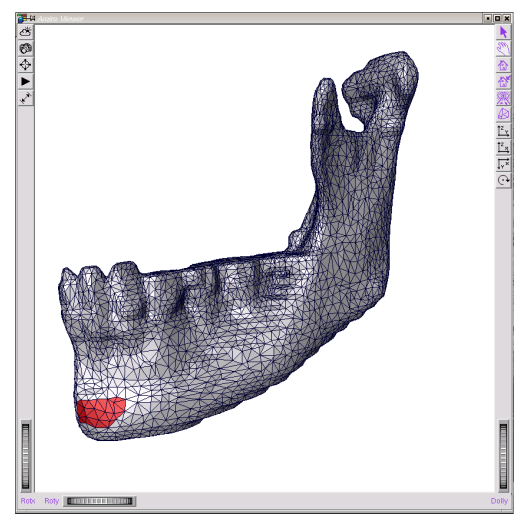

FiguRE 2. Initial mesh with distractor attachment area (dark) for Dirichlet condition.

\subsection{Boundary conditions}

Displacement is inhibited in $x$ direction, according to the above symmetry assumption, in the median plane $\{x=0\}$, i.e. on the left boundary of the callus region in the model. On the area of attachment of the distractor, see Fig. 2, the displacement is prescribed as $1.0 \mathrm{~mm}$ in $x$-direction and $0.0 \mathrm{~mm}$ in $y$ and $z$.

\subsection{Constitutive laws}

Two regions of different materials are considered, representing the original bone and the newly-formed callus, respectively. Both materials are assumed as isotropic and linearly elastic, thus characterized by their Young's moduli $E_{b}, E_{c}$ and Poisson's coefficients $\nu_{b}, \nu_{c}$. The values $E_{b}=15 G P a$ and $\nu_{b}=\nu_{c} 0.3$ have been set as constants, while we define an $x$-dependent function $E_{c}(x ; n)$ for the fracture callus in each step $(n$ being the step number), as

$$
E_{c}(x ; n)=E_{C}(n)-\frac{x}{x_{S}}\left(E_{C}(n)-E_{C 0}\right),
$$

where $x_{S}$ is the (i.e. a representatively chosen) $x$-coordinate of the interface between callus and bone after the $(n-1)$ st step, and $E_{C}(n)$ is the assumed maximal value of the Young's modulus in callus after $(n-1)$ time units corresponding to one distraction step (e.g. days), modeled by linear interpolation between new callus (with Young's modulus $E_{C 0}$ ) and bone as

$$
E_{C}(n)=E_{C 0}+\frac{n}{N}\left(E_{b}-E_{C 0}\right)
$$

where $N$ is the number of steps it takes for the callus to transform into bone.

\section{Numerical implementation}

The geometry for the preoperative situation was gained from individual patient computed tomography data. The bone regions were identified by half-automatical segmentation using the graphics software AMIRA, which also generates a triangulation of all the interfaces between materials in the segmented data set. This initial surface grid must be simplified and further be manually edited to improve its characteristics such as the maximum ratio of the triangles' circumcircle-incircle diameters. Finally, the tetrahedral grid is generated from the surface grid by an AMIRA [7] module, and both grids are used to produce the geometry-defining files for the finite element simulations.

We use linear elements on a tetrahedral grid for the spatial discretisation of the elliptic boundary value problem and perform $N$ successive distraction steps. In each step we calculate the displacement of the mandible and 
callus and represent the deformed geometry by a new tetrahedral grid, consisting of one region for bone and one for the fracture callus. This grid then used as reference configuration for the next distraction step.

\subsection{The grid for the callus region}

Since there were no CT data available for the postoperative states, the geometry for the new material was generated artificially. We assumed that the callus would develop in the region between the original and the deformed positions of the cut surface. These two surface patches were connected in a cylinder-like shape by additional triangles, and the resulting callus region surface (grid) was filled with tetrahedra by the AMIRA module TetraGen. The unions of the regions bone and callus then could be represented by one volume as well as one surface grid, as necessary for the next finite element simulation step.

\section{Results}

\subsection{Displacements}

The simulations show that the condylar head does not move parallel with the distractor but rather rotates about a vertical axis, due to the fixation of the model in the plane of symmetry and to the fact that the displacement by the distractor is enforced on the anterior surface of the mandible; see Fig. 3.

The mandible half in the third quarter of Fig. 3 that seems to have been rotated in the $(y, z)$-plane comes from a variation of the boundary conditions, where Dirichlet data on the interface between distractor and mandible have been prescribed only for the displacement in $x$ direction. (The simulation still yields one solution in this case.) Since the only other boundary condition present, the one on the cut surface, leaves translation in the $(y, z)$-plane free, the restrictions in $y$ and $z$ directions on the distractor surface cannot be expected to make an essential difference for the deformation.

\subsection{Stresses}

The stress tensors were calculated from the displacements. The reaction forces caused by the distractor in the area of its attachment can then be determined. They are for the first 5 steps as follows:

\begin{tabular}{|c||r|r|r|r|r|}
\hline Step & \multicolumn{1}{|c|}{1} & \multicolumn{1}{c|}{2} & \multicolumn{1}{c|}{3} & \multicolumn{1}{c|}{4} & \multicolumn{1}{c|}{5} \\
\hline \hline$F_{x}\left[\mathrm{~N} / \mathrm{cm}^{2}\right]$ & 30469.40 & 28192.00 & 26901.50 & 25991.00 & 25252.20 \\
\hline$F_{y}\left[\mathrm{~N} / \mathrm{cm}^{2}\right]$ & 7126.11 & 6339.72 & 5567.30 & 4778.78 & 4019.98 \\
\hline$F_{z}\left[\mathrm{~N} / \mathrm{cm}^{2}\right]$ & 1650.98 & 1615.96 & 1531.63 & 1445.84 & 1394.51 \\
\hline
\end{tabular}

It appears that the reactions are decreasing during the distraction process. The von Mises stresses are shown in Fig. 4 for the first and fifth steps. They show a pronounced concentration near the distractor area: the values in the light areas in the two top figures (colorscale from $0 P a$ to $20 P a$ and above) are all above $10 P a$, while the different colorscale in the bottom figures ( $0 \mathrm{~Pa}$ to $0.2 \cdot 10^{-4} \mathrm{~Pa}$ ) shows that the von Mises stresses in the vertical part of the mandible are all below $0.0001 \mathrm{~Pa}$. - Although the crudeness of the mesh in the callus region makes any interpretation of the results there somewhat arbitrary, we point out that the stresses are obviously much smaller than in the neighbouring bone, while the strains are larger than in the bone. This corresponds to the considerably lower Young's modulus for the callus material.

\section{Discussion}

The calculated displacements of the mandible with a free boundary near the condylar head, when compared to the position of the temporal bone, make it obvious that the situation at the temporomandibular joint plays an essential role for the median mandibular distraction - and vice versa. Its biomechanical modeling is, hoewever, a very complex task.

Aside from the distraction problem, finite element studies concerning the TMJ have been done since some time. 


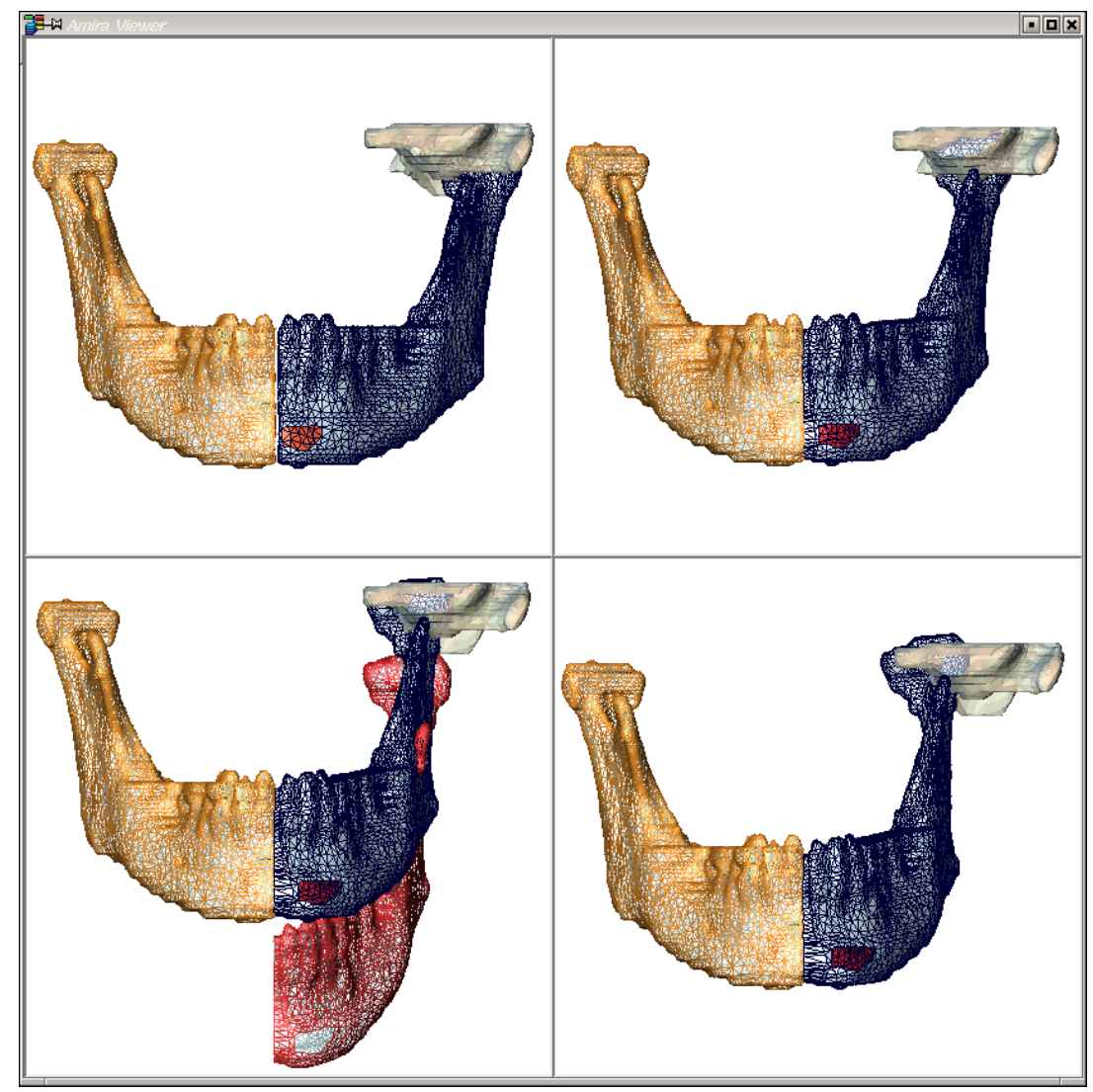

Figure 3. Displacement of the left mandible half after 1, 3, 5, 6 steps. For comparison: Unchanged positions of the right half and of the left temporal bone

Models in tow dimensions were published by Chen and $\mathrm{Xu}$ [3] in 1994 and by DeVocht et. al [5] in 1996; the paper by Koolstra et al. [2] is obviously the first that allowed for unrestricted sliding of a deformable articular disc between temporal bone and condylar head in three dimensions. A classical 3D model including the whole mandible, discus and part of the temporal bone is Korioth et al.'s [4], but it represents the interfaces as mere material boundaries, without the possibility of sliding. This is not surprising, since the contact problem in 3D with a complex geometry is still difficult to treat numerically. (All the three studies $[2,3,5]$ used commercial software packages for this purpose, or for the $2 \mathrm{D}$ problem, respectively.)

The idea behind the constitutive law (1) for the developing callus was that there was for each timestep a correlation, assumed linear, between the $x$-position and the age of the callus. According to (1), the newest material should be located near the bone ends. This view is supported by the finding in post-operative CT data that after a healing period a region of high Hounsfield values can be found in the middle of the former gap. On the other hand, a recent internet publication [8] describing the process of osteogenic distraction says that the tension- stimulated bone formation advances along the collagen fibers, starting from the surfaces of the existing bone ends and progressing towards the fibrous interzone. From this it seems that the mineralization should on the contrary be highest near the original bone.

Another possibility for the model is to begin with a homogeneous value for each material and then to modify it iteratively depending on the calculated mechanical quantities, - taking them as a mechanical stimulus for tissue adaptation (see, e.g., [1] for fracture healing without distraction). 


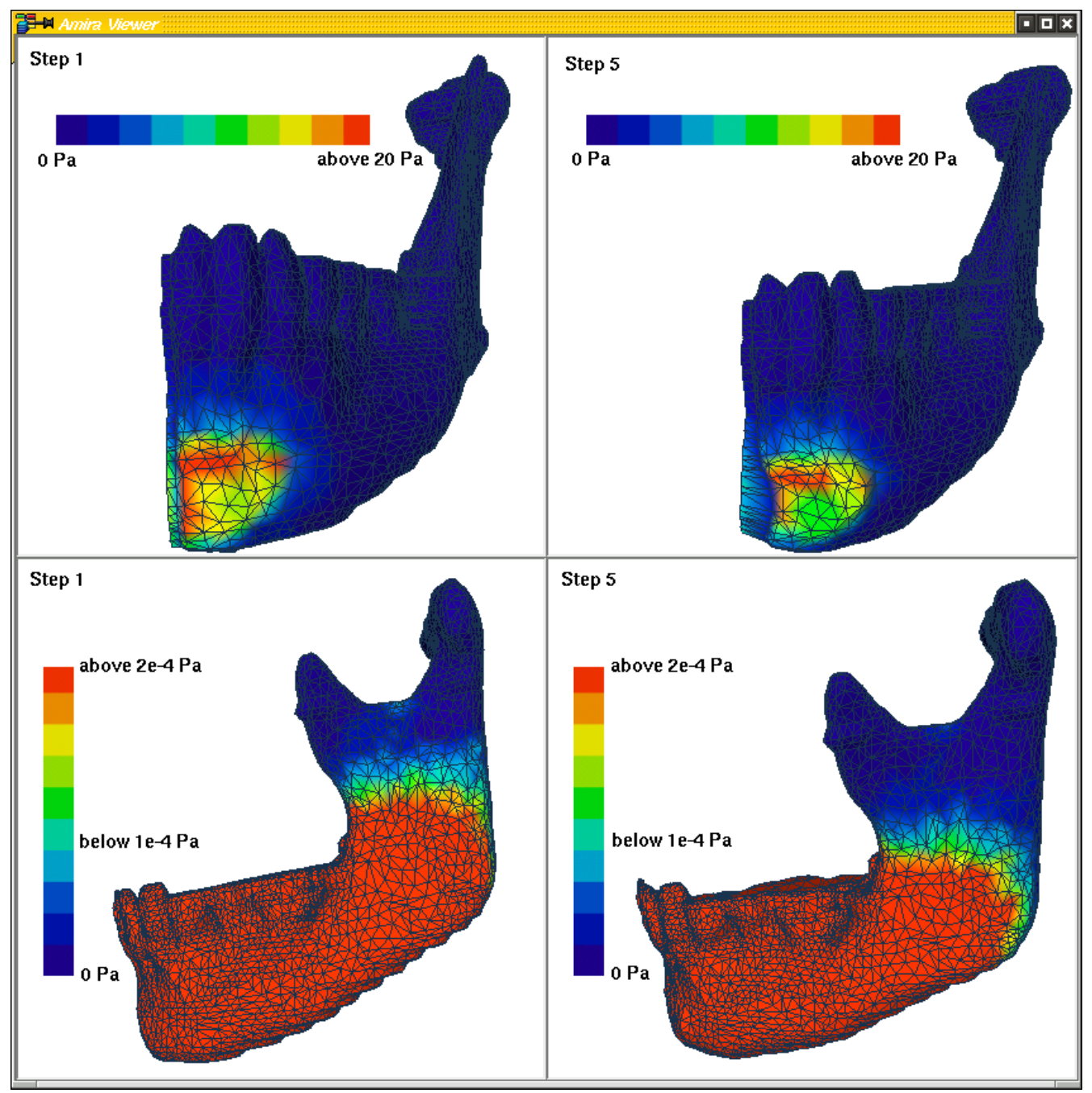

Figure 4. Von Mises stresses after 1 step (left) and after 5 steps (right).

\section{REFERENCES}

[1] C. Ament: Mathematische Modellbildung und Simulation der Knochenheilung. Fortschr.-Berichte VDI Series 17 , No. 158. Düsseldorf: VDI Verlag, 1997.

[2] M.Beek, J.H.Koolstra, L.J.van Ruijven, T.M.van Eijden: Three-dimensional finite element analysis of the human temporomandibular joint disc. J Biomech 2000 Mar;33(3):307-16.

[3] J.Chen, L. Xu: A Finite Element analysis of the Human Temporomandibular Joint. J. Biomed. Engineering 1994 Nov.; 116 : 401-407.

[4] T.W.P.Korioth, D.P.Romilly, A.G.Hannam: Three-dimensional Finite Element Stress Analysis of the Dentate Human Mandible. American Journal of Physical Anthropology 1992, 88: 69-96.

[5] J.D.DeVocht, V.K.Goel, D.L.Zeitler, D.Lew: A Study of the Control of Disc Movement Within the Temporomandibular Joint Using the Finite Element Technique. J. Oral Maxillofac. Surgery 1996, 54: 1431-1437.

[6] J. Sobotta, Atlas der Anatomie des Menschen, Vol. 1. München; Wien; Baltimore: Urban \& Schwarzenberg, 1993.

[7] D.Stalling, M.Zöckler, H.-Chr.Hege: AMIRA - Advanced Visualization, Data Analysis and Geometry Reconstruction. http://www. amira.zib.de

[8] J. Wheeler et al.: The miracle of growing bone: Craniofacial distraction osteogenesis. Currents: Physician-To-Physician Clinical Resource, Vol. 3, No. 2 (2002), www.uihealthcare.com/news/currents/vol3issue2 